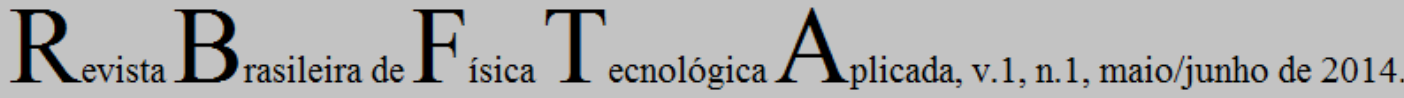

\section{Estudo do Efeito do Transporte de Massa nas Curvas de Polarização Potenciodinâmica em Ligas de Alumínio da Série 7XXX de aplicação Aeronáutica}

Estudio sobre el efecto del transporte masivo en las curvas de polarización potenciodinámicas para aleaciones de Aluminio serie 7XXX de aplicación aeronáutica

\section{The Study of Mass Transport Phenomena on the Potentiodynamic Polarization Curves in 7XXX series Aluminum Alloys for the Aircraft Construction}

\begin{abstract}
Jéferson Aparecido Moreto ${ }^{1}$, José Neto Costa Mota, Luciana Sgarbi Rossino, Waldek Wladimir Bose Filho, Marcelo Ferreira Moreira, João Carlos Salvador Fernandes, Luís Augusto da Rocha
\end{abstract}

Resumo: As ligas de Alumínio ( $\mathrm{Al}$ ) são largamente utilizadas pela indústria aeronáutica, devido à sua baixa densidade, alta resistência mecânica e boa resistência à corrosão. A sua resistência à corrosão é atribuída à película de óxido $\left(\mathrm{Al}_{2} \mathrm{O}_{3}\right)$ que se forma espontaneamente na superfície do material. No entanto, na presença de íons agressivos, as ligas de $\mathrm{Al}$ estão sujeitas ao processo de corrosão localizada. $\mathrm{O}$ comportamento eletroquímico das ligas AA7050-T7451 e AA7081-T73511 utilizadas pela indústria aeronáutica foi investigado sob as condições de transporte de massa controlado através de um eletrodo cilíndrico rotatório em meio de uma solução de $0,6 \mathrm{M} \mathrm{NaCl}$. A densidade de corrente teórica foi determinada através da equação de Kouteki - Levich. Os resultados confirmam que o intermetálico $\mathrm{Al}_{7} \mathrm{Cu}_{2} \mathrm{Fe}$ atua como cátodo preferencial causando o acoplamento galvânico e a dissolução da matriz de $\mathrm{Al}$ em torno dele.

Palavras-chave: corrosão; ligas de alumínio; eletrodo cilíndrico rotatório; meio salino.

Resumen: Las aleaciones de Aluminio (Al) son ampliamente utilizadas en la industria aeronáutica, debido a su baja densidad, alta resistência mecánica y gran resistencia a la corrosión. Su resistencia a la corrosión se atribuye $a$ la película de óxido $\left(\mathrm{Al}_{2} \mathrm{O}_{3}\right)$ que se forma espontáneamente en la superficie del material. Sin embrago, en presencia de iones agresivos, las aleaciones de Al estánsujeta a procesos de corrosión localizada. El comportamiento electroquímico de las aleaciones AA7050-T7451 y AA7081-T73511 utilizadas por la industria aeronáutica fue investigado bajo las condiciones de transporte masivo controlado a través de un electrodo cilíndrico giratorio en el interior de una solución de $0,6 \mathrm{M} \mathrm{NaCl}$. La densidad de la corriente eléctrica fue determinada a través de a ecuación de Kouteki - Levich. Los resultados obtenidos confirman que el intermetálico $\mathrm{Al}_{7} \mathrm{Cu}_{2} \mathrm{Fe}$ actúa como cátodo preferente, provocando el par galvánico y la disolución de la matriz de $\mathrm{Al}$ a su alrededor.

Palabras clave: corrosión; aleaciones de aluminio; electrodo cilíndrico giratório; medio salino.

\begin{abstract}
Aluminum Alloys are widely used as structural materials in the aerospace industry due to low weight, high mechanical strength and enduring corrosion resistance. Their resistance to corrosion is attributed to the rapidly formed stable oxide film $\left(\mathrm{Al}_{2} \mathrm{O}_{3}\right)$ which spontaneously forms itself on the surface of the material. However, in the presence of aggressive ions, such as halide, Aluminum Alloys are subject to a localized process of corrosion. The electrochemical behavior of 7081-T73511 and 7050-T7451 Aluminum Alloys employed in the aerospace industry was investigated using a $0.6 \mathrm{M} \mathrm{NaCl}$ solution under the conditions of a controlled mass transport employing a rotating disk electrode. The theoretical limiting current density was determined by the Kouteki-Levich equation. The results confirmed that the inter-metallic $\mathrm{Al}_{7} \mathrm{Cu}_{2} \mathrm{Fe}$ acts as preferential cathode generating the galvanic coupling and the dissolution of the Aluminum matrix around it.
\end{abstract}

Keywords: corrosion; aluminum alloys; rotating cylindrical electrode; saline environment.

${ }^{1}$ Professor do Instituto Federal Goiano, câmpus Morrinhos - Rodovia BR-153, Km 633, Zona Rural, Caixa Postal 92, CEP: 75659-000 Morrinhos, Goiás, Brasil.e-mail : Jeferson.moreto@ifgoiano.edu.br

RBFTA, v.1, n.1, maio/junho de 2014, p. 23 


\section{Introdução}

O alumínio (Al) é o metal não ferroso mais consumido em todo o mundo. Já era conhecido no tempo dos antecedentes romanos e utilizado para fins médicos e, mesmo com o crescimento das oportunidades no mercado para outros materiais, o Al continua a competir com vários materiais, pois oferece baixo custo e outras vantagens competitivas (TOTTEN e MACKENZIE, 2003).

As ligas de Al de elevada resistência mecânica são caracterizadas por uma microestrutura complexa e que tem sua origem na adição de elementos de liga e de realização de tratamentos térmicos e/ou termomecânicos para a obtenção de propriedades mecânicas adequadas às condições de uso.

O processamento das ligas de Al, que objetiva aumentar suas propriedades mecânicas, gera a precipitação de partículas intermetálicas (IMs) com dimensões típicas entre $2 \mu \mathrm{m}$ (KLOET, HASSEL e STRATMANN, 2005) e $30 \mu \mathrm{m}$ (GUILLAUMIN e MANKOWSKI, 1999) além de outras partículas com dimensões submicrométricas (DAVIS, 1993). A presença de partículas IMs provoca processos de corrosão localizada (pites), corrosão intergranular e esfoliação.

A corrosão localizada associada à presença de IMs é o ponto de partida para diversos tipos de corrosão que atingem as ligas de Al de elevada resistência mecânica. Estudos de corrosão localizada para as ligas da série 7XXX são menos abundantes (De WIT, 2004; BIRBILIS, CAVANAUGH e BUCHHEIT, 2006; WLOKA e VIRTANEN, 2007). A maioria dos trabalhos utilizando ligas da série 7XXX são dedicados ao estudo da corrosão associada às solicitações mecânicas, como por exemplo, corrosão sob tensão (WANG e MA, 2009).

As ligas da série 7XXX (Al-Zn-Cu-Mg) apresentam o zinco (Zn) como principal elemento de liga, em teores de $\mathrm{Zn}$ entre $1 \%$ e $8 \%$. Adições de magnésio $(\mathrm{Mg})$, cobre $(\mathrm{Cu})$ e cromo (Cr) são comuns e resultam em ligas tratáveis termicamente de elevada resistência mecânica. As adições de $\mathrm{Zn}, \mathrm{Cu}$ e Mg promovem a precipitação coerente e semi-coerente das fases $\theta^{\prime}\left(\mathrm{Al}_{2} \mathrm{Cu}\right)$ e $\eta^{\prime}\left(\mathrm{Zn}_{2} \mathrm{Mg}\right)$ que são responsáveis pela alta resistência mecânica nestas ligas.

$\mathrm{O}$ alto teor de $\mathrm{Zn}$ presente nestas ligas as torna mais anódicas em relação ao Al puro (DAVIS, 1993). As ligas AA7081-T73511 e AA7050-T7451 foram desenvolvidas para combinar alta resistência mecânica, baixa densidade e boa resistência à fadiga. A liga AA7081-T73511 foi desenvolvida recentemente e poucos estudos são encontrados na literatura (MORETO, 2012; CHEMIN et al, 2013).

RBFTA, v.1, n.1, maio/junho de 2014, p. 24 
A liga AA7081-T73511 produzida em forma de chapa oferece uma resistência superior combinada com maior tenacidade à fratura quando comparada às ligas convencionais da série 7XXX. Com densidade aproximada à das ligas de Al-Li e excelentes propriedades em resistência à fadiga, desgaste e soldabilidade são utilizadas em aplicações estruturais (MORETO, 2012).

A liga AA7050-T7451 foi desenvolvida pela ALCOA em 1970. Os elementos Zn e $\mathrm{Mg}$ aumentam a formação de precipitados que levam ao endurecimento por precipitação e o $\mathrm{Cu}$ melhora a resistência mecânica. Além disso, a liga contém $\mathrm{Zn}$, que forma dispersóides $\mathrm{Al}_{3} \mathrm{Zn}$ que, no caso, retardam a recristalização.

$\mathrm{O} \mathrm{Fe}$ e o $\mathrm{Si}$ são tidos como impurezas, formando fases intermetálicas com o $\mathrm{Al}$ e outros elementos ligantes, por exemplo, $\mathrm{Al}_{7} \mathrm{Cu}_{2} \mathrm{Fe}$ e $\mathrm{MgSi}$. De acordo com Birbilis, Cavanauch e Buchheit (2006) as partículas $\mathrm{Al}_{7} \mathrm{Cu}_{2} \mathrm{Fe}$ são maiores, quando comparada a outros IMs encontrados nas ligas da série 7XXX.

Nesse contexto, este trabalho tem por objetivo avaliar o comportamento eletroquímico das ligas AA7081-T73511 e AA7050-T7451utilizadas pela indústria aeronáutica sob as condições de transporte de massa controlado através de um eletrodo de disco rotatório em meio de uma solução salina de 3,5\% NaCl.

\section{Experimental}

\subsection{Materiais}

Os materiais utilizados no presente trabalho foram duas ligas de alumínio da série 7XXX com a designação 7081 e 7050, nas condições T73511 e T7451, de aplicação estrutural. A Tabela 1 apresenta as composições químicas (\% em peso) das ligas estudadas. Os valores entre parênteses referem-se a valores nominais especificados pelo fabricante.

Todos os elementos analisados estão dentro dos padrões nominais estabelecidos pela norma ASM 4341, relacionada à liga AA7050-T7451. No que diz respeito à liga AA7081T73511 os valores encontrados estão dentro dos limites estipulados pelo fabricante (ALERIS). 
Tabela 1: Composição química nominal (\% em peso) das ligas AA7081-T73511 e AA7050-T7451

\begin{tabular}{|c|c|c|}
\hline Elemento & AA7050-T7451 & AA7081-T73511 \\
\hline $\mathrm{Cu}$ & $2,25(2,0-2,6)$ & $1,69(1,2-1,8)$ \\
\hline $\mathrm{Li}$ & - & - \\
\hline $\mathrm{Si}$ & 0,04 (0,12máx) & 0,02 (0,12máx) \\
\hline $\mathrm{Fe}$ & 0,05 (0,15máx) & $0,04(0,15)$ \\
\hline $\mathrm{Mg}$ & $1,896(1,9-2,6)$ & $1,935(1,8-2,2)$ \\
\hline Mn & 0,01 (0,1máx) & ND (0,25 máx) \\
\hline $\mathrm{Ti}$ & 0,03 (0,06máx) & - \\
\hline $\mathrm{Ag}$ & - & - \\
\hline $\mathrm{Zr}$ & $0,10(0,08-0,15)$ & - \\
\hline $\mathrm{Zn}$ & $6,02(5,7-6,7)$ & $7,24(6,9-7,5)$ \\
\hline $\mathrm{Cr}$ & 0,01 (0,04 máx) & ND (0,04 máx) \\
\hline $\mathrm{Al}$ & Balanço & Balanço \\
\hline
\end{tabular}

\subsection{Ensaios de corrosão}

No presente trabalho dois ensaios de corrosão foram realizados, a citar: potencial de circuito aberto e polarização potenciodinâmica em eletrodo cilíndrico rotatório.

Os ensaios de potencial de circuito aberto foram realizados com o objetivo de caracterizar a tendência termodinâmica para a corrosão das ligas de Al. O potencial de corrosão $\left(\mathrm{E}_{\text {corr }}\right)$ das amostras foi monitorado durante $600 \mathrm{~s}$ precedente aos ensaios de polarização potenciodinâmica. As curvas de polarização potenciodinâmica em eletrodo cilíndrico rotatório foram realizadas com a finalidade de oferecer um panorama sobre a cinética eletroquímica em função do potencial e as condições do transporte de massa. Os ensaios de potencial de circuito aberto e polarização potenciodinâmica em eletrodo cilíndrico rotatório foram realizados utilizando-se um potenciostato/galvanostato GAMRY 600 acoplado a um microcomputador para o controle e processamento dos dados. Para esse ensaio utilizou-se uma célula eletroquímica contendo um único compartimento de três eletrodos, sendo eles: eletrodo de trabalho (ET) constituído das ligas estudadas (AA7081-T73511 e AA7050-T7451), contra - eletrodo (CE) de platina (Wire B35M 110 - radiometer analytical) 
e eletrodo de referência (ER) de calomelano saturado $\mathrm{Hg} / \mathrm{Hg}_{2} \mathrm{Cl}_{2}, \mathrm{KCl}_{\text {sat }}(\mathrm{B} 20 \mathrm{~B} 110$ radiometer analytical). A solução utilizada nos experimentos foi $0,6 \mathrm{M} \mathrm{NaCl}$.

Os eletrodos cilíndricos rotatórios foram usinados em forma cilíndrica com $6 \mathrm{~mm}$ de diâmetro e embutidos em resina epóxi deixando exposta apenas uma superfície plana $(0,283$ $\mathrm{cm}^{2}$ ). As amostras foram polidas mecanicamente até 2400 mesh em lixas de carbeto de silício (SiC). A Figura 1 apresenta a forma dos eletrodos utilizados nos ensaios de polarização potenciodinâmica. É possível verificar um fluxo laminar próximo da superfície do eletrodo.

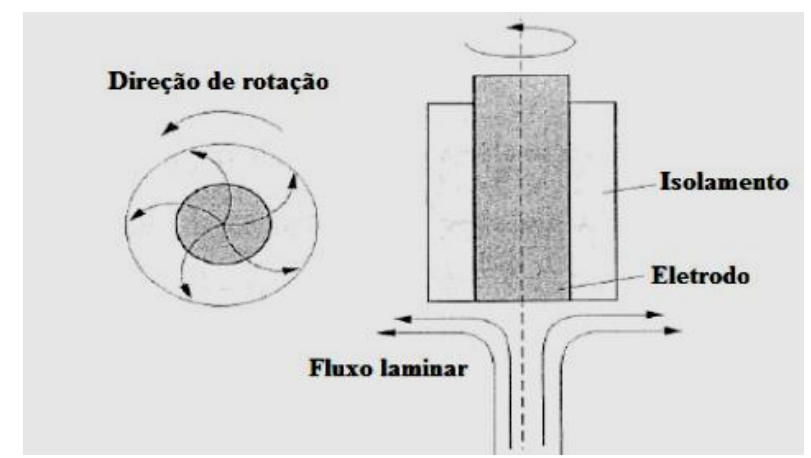

Figura 1: Forma dos eletrodos rotatórios utilizados nos ensaios de polarização potenciodinâmica Fonte: Adaptado de Landolt (2007)

A Figura 2 apresenta o arranjo experimental utilizado nos ensaios de polarização em eletrodo rotatório.

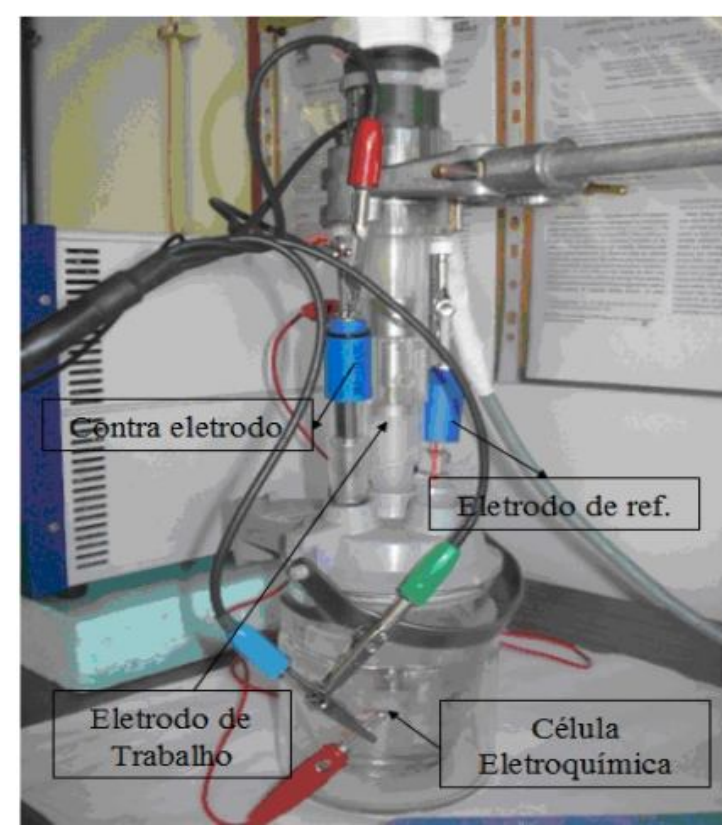

Figura 2: Arranjo experimental mostrando a célula eletroquímica e os eletrodos de trabalho, referência e contra-eletrodo.

Fonte: Próprio autor 
Inicialmente as medidas de polarização foram feitas a partir de um potencial catódico de $-1,4 \mathrm{~V}$, com uma velocidade de varredura de $0,5 \mathrm{mV} / \mathrm{s}$. Entretanto, houve uma variação considerável no pH da solução. Dessa maneira optou-se por realizar os ensaios a partir de um potencial de $-0,8 \mathrm{~V}$ de forma a minimizar as alterações superficiais possivelmente induzidas por processos de corrosão anódicos. $\mathrm{O}$ pH foi medido antes e depois de cada ensaio e não houve variação. As taxas de rotação selecionadas para este estudo foram: 0, 100, 400, 800 e $1200 \mathrm{rpm}$. Os experimentos eletroquímicos foram conduzidos à temperatura de $25 \pm 1{ }^{\circ} \mathrm{C}$.

\section{Resultados e Discussão}

As Figuras 3(a), 3(b) e 3(c) apresentam as micrografias obtidas via microscopia eletrônica de varredura (MEV) das ligas AA7081-T73511 e AA7050-T7451 antes dos ensaios de corrosão. É possível verificar que a liga AA7081-T73511é constituída por elementos uniformemente distribuídos e por fases tais como: incrustações devido às impurezas e precipitados.

A liga AA7081-T73511 apresenta perfurações e/ou frestas quando submetida a tratamento superficial mecânico. De acordo com Balducci et al (2001) o desprendimento de material ocorre devido à diferença de dureza entre as partículas intermetálicas e a matriz e/ou a partir da dissolução parcial das fases ou da matriz na interface, dependendo se as fases são anódicas ou catódicas respectivamente. $\mathrm{O}$ intermetálico (IM) $\mathrm{Al}_{7} \mathrm{Cu}_{2} \mathrm{Fe}$ foi determinado na liga AA7050-T7451 por meio de espectroscopia de energia dispersiva de raios-X (EDX). Segundo os autores Chemin et al (2013) o $\mathrm{IM} \mathrm{Al}_{7} \mathrm{Cu}_{2} \mathrm{Fe}$ resulta do processo de solidificação, apresentando uma morfologia alongada e espaços vazios, vide Figuras 3(b) e 3(c).

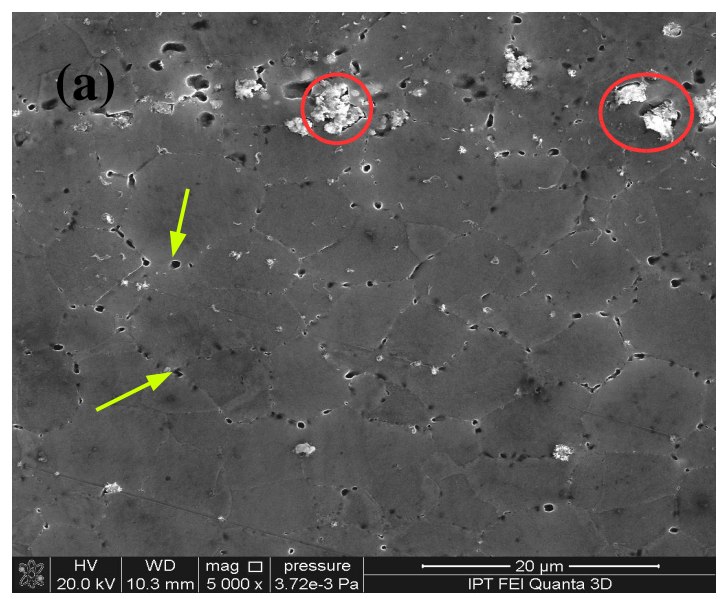



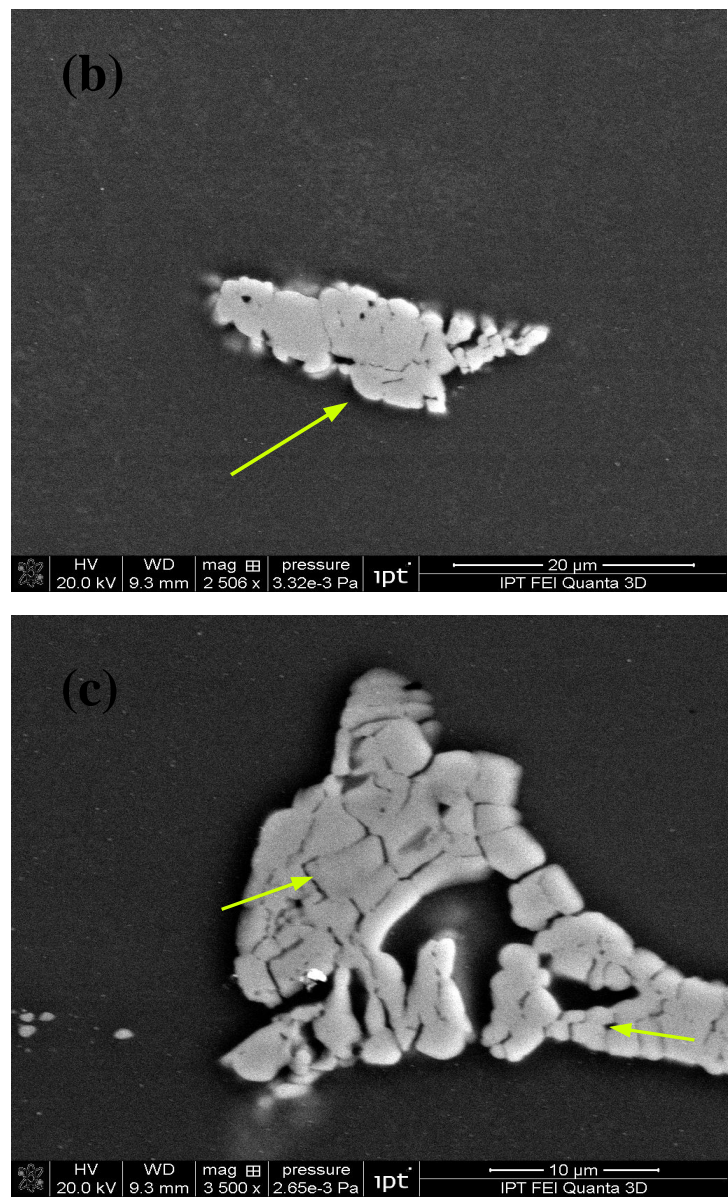

Figura 3: Microestrutura das ligas AA7081-T73511 e AA7050-T7451 viaMEV: (a) setas amarelas mostrando as incrustações/impurezas e os círculos vermelhos os precipitados para a liga AA7081-T73511;

(b) $\mathrm{IM}_{\mathrm{Al}} \mathrm{Cu}_{2} \mathrm{Fe}$ na superfície da liga AA7050-T7451 e (c) IM na superfície da liga AA7081-T73511.

A Figura 4 apresenta as curvas de potencial de circuito aberto para as ligas AA7081T73511 e AA7050-T7451. As oscilações que acompanham as medidas possivelmente são devidas a pites que contribuem para a descontinuidade do filme. A corrosão por pite é causada pela ação localizada de íons agressivos, na sua maioria $\mathrm{Cl}^{-}$em um meio no qual o filme passivante não é estável (NISANCIOGLU, 1992). Nesse contexto, é possível supor que a dependência do potencial com o tempo (E vs. ECS - t) está vinculada com a variação da razão de áreas coberta/descoberta e/ou catódica/anódica. 


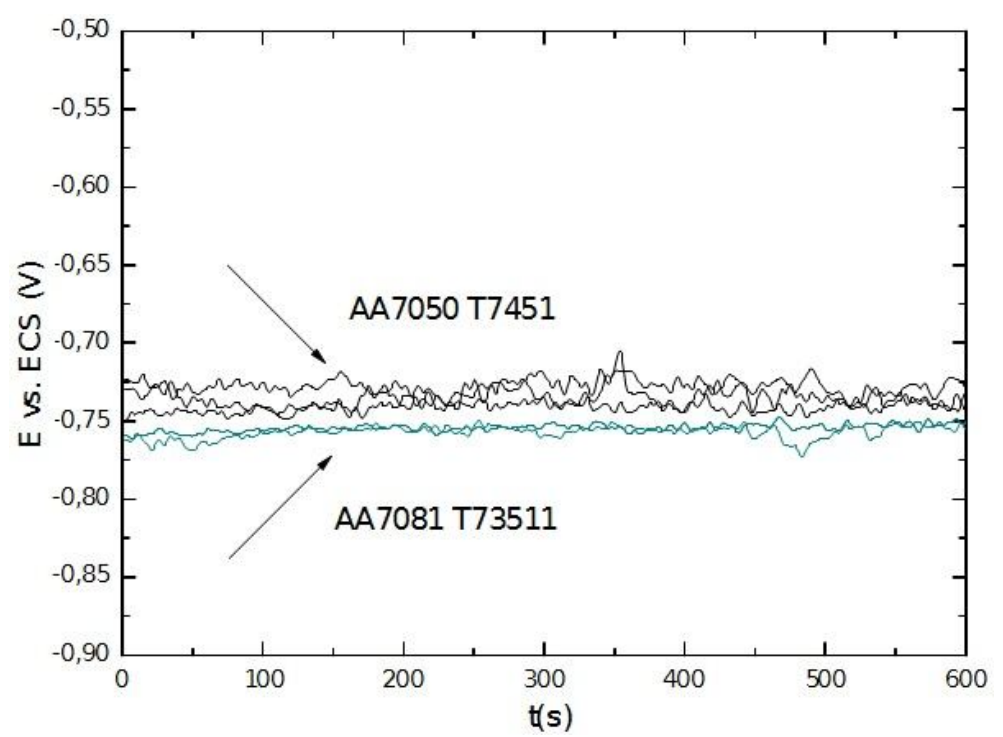

Figura 4: Curvas de potencial de circuito aberto para as ligas AA7081-T73511 e AA7050-T7451 em solução 0,6 M NaCl.

As Figuras 5 e 6 apresentam as curvas de polarização potenciodinâmica em diferentes velocidades de rotação para as ligas AA7081-T73511 e AA7050-T7451 em solução de 0,6 M de $\mathrm{NaCl}$. Por meio das curvas de polarização potenciodinâmica (eletrodo estático) é possível verificar que ambas as ligas sofrem corrosão por pite $\left(\mathrm{E}_{\text {pite }}\right)$ no potencial de corrosão $\left(\mathrm{E}_{\text {corr }}\right)$. Os valores da densidade de corrente de corrosão $\left(i_{c o r r}\right)$ são similares, indicando que a área ocupada por sítios catódicos é praticamente a mesma. A região catódica não foi afetada pela rotação do eletrodo.

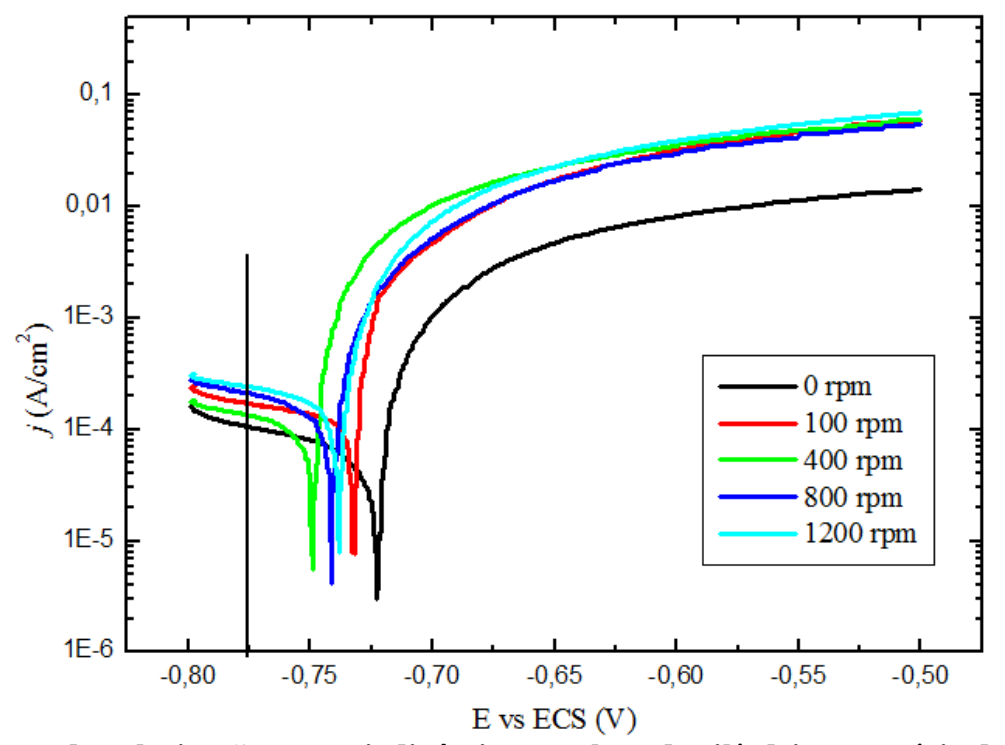

Figura 5: Curvas de polarização potenciodinâmica em eletrodo cilíndrico rotatório da liga AA7081T73511 reunindo as diferentes velocidades de rotação, solução $0,6 \mathrm{M} \mathrm{NaCl}$. 


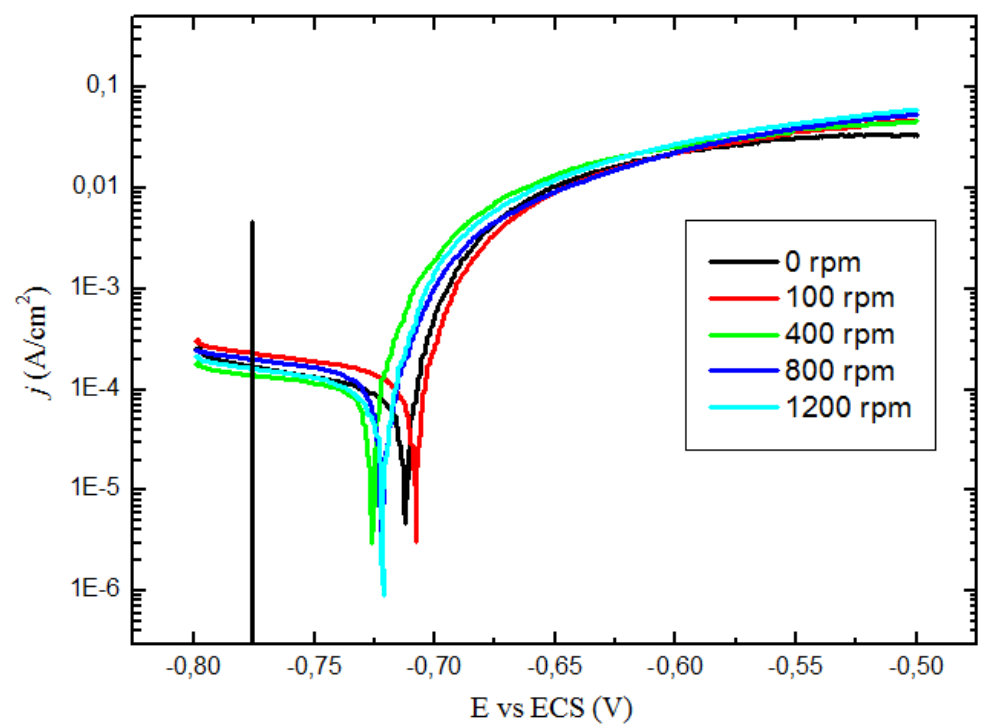

Figura 6: Curvas de polarização potenciodinâmica em eletrodo cilíndrico rotatório da liga AA7050-T7451 reunindo as diferentes velocidades de rotação, solução 0,6 M NaCl.

As Figuras 7 e 8 apresentam as micrografias obtidas após os ensaios de polarização potenciodinâmica em eletrodo cilíndrico rotatório em meio de solução de $0,6 \mathrm{M} \mathrm{NaCl}$. Por meio das micrografias é possível perceber uma dissolução preferencial na interface do IM com a matriz (Figura 7). Isto está de acordo com o mecanismo de corrosão localizada e o acoplamento galvânico entre o IM e a vizinhança (material base). O IM $\mathrm{Al}_{7} \mathrm{Cu}_{2} \mathrm{Fe}$ foi identificado em ambas as ligas estudadas via espectroscopia de energia dispersiva de raios- $\mathrm{X}$ $(\mathrm{EDX})$

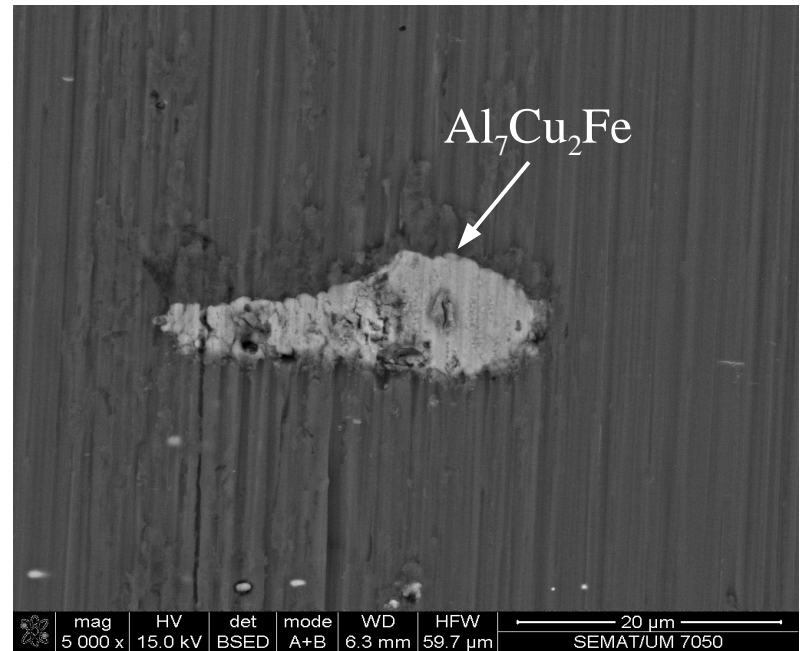

Figura 7: Microestrutura da liga AA7050-T7451 após os ensaios de polarização potenciodinâmica em eletrodo cilíndrico rotatório. 


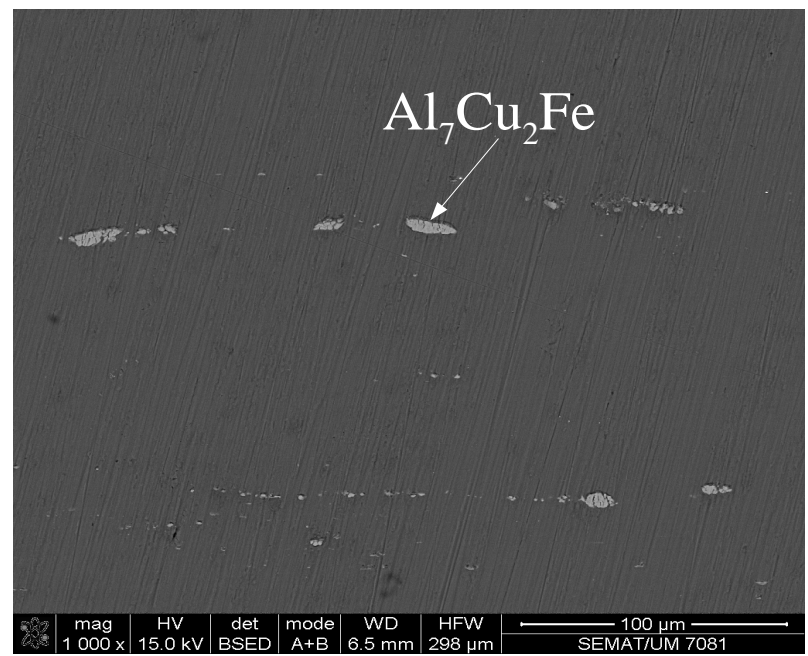

Figura 8: Microestrutura da liga AA7081-T73511 após os ensaios de polarização potenciodinâmica em eletrodo cilíndrico rotatório.

De Wit (2004) identificou por meio de MEV/EDX para a liga AA7349 quatro partículas intermetálicas principais, também de dimensões micrométricas $(1 \mu \mathrm{m}-10 \mu \mathrm{m})$, a saber: $\mathrm{Al}_{7} \mathrm{Cu}_{2} \mathrm{Fe},(\mathrm{Al}, \mathrm{Cu})_{6}(\mathrm{Fe}, \mathrm{Cu}, \mathrm{Mn}), \mathrm{Mg}_{2} \mathrm{Si}$ e $(\mathrm{Al}, \mathrm{Cu}, \mathrm{Fe}, \mathrm{Mn}, \mathrm{Si}) . \mathrm{O}$ autor ainda comprovou que para a liga AA7075, as partículas intermetálicas mais abundantes na microestrutura são aquelas que contêm Fe e Cu. De acordo com o autor De Wit (2004), as ligas AA7349 e AA7075 apresentam os intermetálicos $\mathrm{Al}_{7} \mathrm{Cu}_{2} \mathrm{Fe}$ com composição química similar com uma relação $(\mathrm{m} / \mathrm{m})$ de $\mathrm{Cu} / \mathrm{Fe}$ entre 1,5 e 2,5.

Segundo os autores Birbilis, Cavanauch e Buchheit (2006) as partículas $\mathrm{Al}_{7} \mathrm{Cu}_{2} \mathrm{Fe}$ possuem caráter catódico em relação à matriz metálica em meio contendo íons $\mathrm{Cl}^{-}$. Wloka e Virtanem (2007) estudaram o comportamento eletroquímico e a corrosão das ligas AA7010 e AA7349 e verificaram que os intermetálicos ricos em $\mathrm{Mg}$ e $\mathrm{Si}\left(\mathrm{Mg}_{2} \mathrm{Si}\right)$ atuam como anodos em relação à matriz metálica, sendo rapidamente dissolvidos em água. No que diz respeito à liga AA7010, os autores verificaram que as partículas $\mathrm{Al}_{7} \mathrm{Cu}_{2} \mathrm{Fe}$ atuam como catodos locais, no qual ocorre a redução preferencial de $\mathrm{O}_{2}$ dissolvido em água, formando íons $\mathrm{OH}^{-}$, resultando no aumento do $\mathrm{pH}$ local.

Pao et al (2003) estudaram a corrosão - fadiga nas ligas AA7075 e AA7050 de grau aeronáutico. De acordo com Pao et al (2003), três tipos de partículas constituintes foram encontradas. A primeira partícula identificada através das técnicas de CBED (Converging beam electron difraction) e SAD (Selected area difraction) como $\mathrm{Al}_{23} \mathrm{CuFe}_{4}$, parâmetro de rede $\mathrm{a}=0,759, \mathrm{~b}=0,6657 \mathrm{e} \mathrm{c}=0,8840 \mathrm{~nm}$ e estrutura cristalina ortorrômbica. O segundo tipo de partícula constituinte contém $\mathrm{Cu} / \mathrm{Mg}$ na forma esférica. Os resultados indicaram que estas partículas provavelmente são $\mathrm{Al}_{2} \mathrm{CuMg}$ possuindo um potencial de corrosão mais negativo se 
comparado com a matriz metálica. A terceira partícula se apresenta em menor quantidade e possui um comportamento eletroquímico neutro frente à matriz metálica. No que diz respeito à liga AA7050, dois tipos de partículas intermetálicas foram encontradas: a primeira foi identificada através de CBED e SAD como tetragonal $\left(\mathrm{Al}_{7} \mathrm{Cu}_{2} \mathrm{Fe}\right)$ com um parâmetro de rede de $\mathrm{a}=0,656 \mathrm{e} \mathrm{c}=1,500 \mathrm{~nm}$, tendo um comportamento catódico em relação à matriz metálica. A segunda partícula encontrada contendo $\mathrm{Si}$ em sua composição. Segundo o autor Nisancioglu (1990) a fase intermetálica $\left(\mathrm{Al}_{7} \mathrm{Cu}_{2} \mathrm{Fe}\right)$ varia com a quantidade de $\mathrm{Fe}$ na liga.

Os mecanismos de corrosão das ligas AA7081-T73511 e AA7050-T7451 podem ser melhores entendidos através do estudo da reação de redução do oxigênio (MARKOVIC et al, 2001; OHTSUKA; NOMURA, 1997; KIM et al, 2006) e essa reação pode ocorrer por meio de 4 elétrons de acordo com a equação 1 ou dois elétrons conforme a equação 2 ou um processo misto, em algum lugar entre estes dois casos limitantes:

$$
\begin{array}{rr}
\mathrm{O}_{2}+2 \mathrm{H}_{2} \mathrm{O}+4 \mathrm{e}^{-} \rightarrow 4 \mathrm{OH}^{-}, & \text {(equação 1) } \\
\mathrm{O}_{2}+\mathrm{H}_{2} \mathrm{O}+2 \mathrm{e}^{-} \rightarrow \mathrm{H}_{2} \mathrm{O}_{2}+2 \mathrm{OH}^{-} & \text {(equação 2) }
\end{array}
$$

As equações 1 e 2 são fortemente influenciadas pelo potencial e envolvem reações elementares. A reação descrita pela equação1 normalmente é controlada por transporte de massa devido à baixa solubilidade do oxigênio na água. A reação de redução da água (equação 2) normalmente é controlada por transferência de carga (LANDOLT, 2007).

Para um melhor entendimento do mecanismo de como as condições de transporte de massa afetam a cinética da região catódica para as ligas AA7081-T73511 e AA7050-T7451, a densidade de corrente catódica $\left(J_{l}\right)$ em $-0,78 \mathrm{~V}$ foi plotada versus a raiz quadrada da taxa de rotação (frequência angular) para o eletrodo cilíndrico rotatório no gráfico de Kouteki Levich. De acordo com Landolt (2007) a cinética da reação de transporte de massa é controlada quando o limite de densidade de corrente varia linearmente com a raiz da taxa de rotação. Os valores teóricos da $J_{l}$ foram determinados por meio da equação de Levich

$$
J_{I}=0,62 n \mathrm{FC}_{\mathrm{B}, \mathrm{b}} \mathrm{D}_{\mathrm{B}}^{2 / 3} \nu^{-1 / 6} \omega^{1 / 2}
$$

onde $\omega$ representa a frequência angular em $\mathrm{rad} / \mathrm{s}\left(f\right.$ é a frequência em $\left.\mathrm{s}^{-1}\right), v$ é a velocidade cinemática em $\mathrm{cm}^{2} \cdot \mathrm{s}^{-1}, \mathrm{D}_{\mathrm{B}}$ o coeficiente de difusão $\mathrm{em} \mathrm{cm}^{2} \cdot \mathrm{s}^{-1}, c_{\mathrm{B}, \mathrm{b}}$ a concentração do bulk em mol.cm ${ }^{-3}, \mathrm{~F}$ a constante de Faraday em C/eq, e $J_{l}$ a densidade de corrente limite em A.cm ${ }^{-2}$ e no número de elétrons em eq/mol. 
De acordo com Landolt (2007) e Vieira et al (2011) os seguintes valores típicos podem ser assumidos: $\mathrm{n}=2 \mathrm{e} / \mathrm{ou} 4 \mathrm{eq} / \mathrm{mol}, \mathrm{F}=96485 \mathrm{C} / \mathrm{eq}, \mathrm{C}_{\mathrm{O} 2}=2,52 \times 10^{-7} \mathrm{~mol} / \mathrm{cm}^{3}, \mathrm{D}_{\mathrm{O} 2}=$ $2,51 \times 10^{-5} \mathrm{~cm}^{2} / \mathrm{s}$ (para o oxigênio em água) e $v=0,01 \mathrm{~cm}^{2} / \mathrm{s}$.

As Figuras 9 e 10 apresentam as curvas de densidade de corrente teórica versus as taxas de rotação (frequência angular em $\mathrm{rad} . \mathrm{s}^{-1}$ ) utilizadas neste trabalho. Por meio dessas figuras é possível perceber que a redução do oxigênio devido a 2 elétrons é mais favorecida para ambas as ligas.

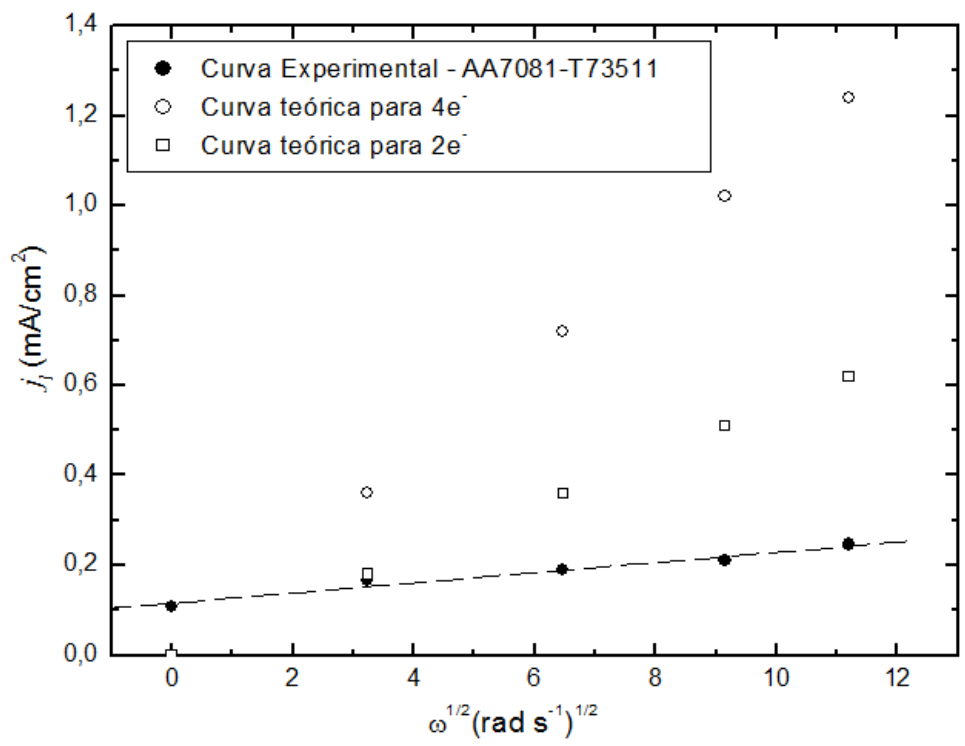

Figura 9: Gráfico de Kouteki - Levich mostrando a densidade de corrente teórica versus a frequência angular para a liga AA7081-T73511.

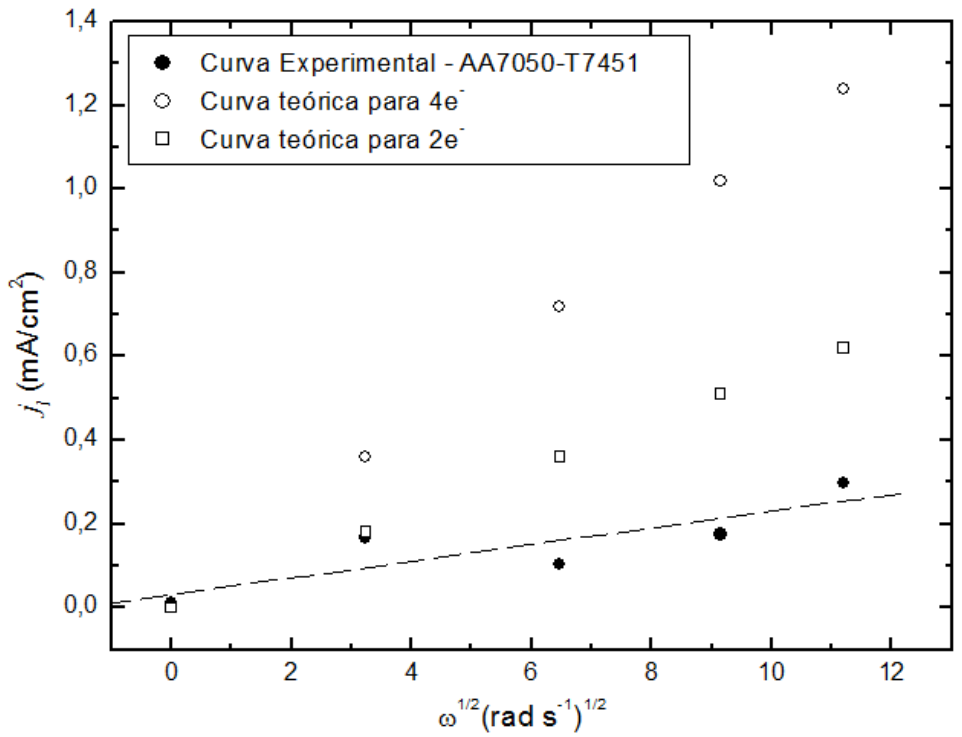

Figura 10: Gráfico de Kouteki - Levich mostrando a densidade de corrente teórica versus a frequência angular para a liga AA7050-T7451. 
O acoplamento galvânico IM-matriz determina a direção de transferência dos elétrons. Dessa maneira, pode-se dizer que os IMs se comportam como cátodos e a matriz como ânodo.

No início, esses precipitados são anódicos em relação ao metal base. Entretanto, durante o processo de corrosão a quantidade de $\mathrm{Cu}$ é aumentada, fazendo com que o potencial dos precipitados se direcione a valores mais positivos, se tornando cátodos em relação ao $\mathrm{Al}$, fazendo com que o metal se dissolva e não mais os IMs.

\section{Conclusão}

O trabalho experimental foi realizado em duas ligas de $\mathrm{Al}$ de grau aeronáutico em solução de 0,6 M NaCl. Os resultados demonstram que as ligas AA7081-T73511 e AA7050T7451 apresentam intermetálicos semelhantes, independente do processamento termomecânico empregado. Através de MEV e EDX foi possível identificar os compostos intermetálicos que são mais ativos na determinação do comportamento de corrosão das ligas AA7081-T73511 e AA7050-T7451.

Em particular, o acoplamento galvânico do intermetálico $\mathrm{Al}_{7} \mathrm{Cu}_{2} \mathrm{Fe}$ com a matriz metálica é considerado um processo muito importante, levando a um processo de dissolução preferencial da matriz em torno das partículas. No que diz respeito às curvas de polarização potenciodinâmica em eletrodo cilíndrico rotatório pode-se perceber que a reação de redução do oxigênio devido a 2 elétrons é mais favorecida para ambas as ligas estudadas.

\section{Referências Bibliográficas}

BALDUCCI, I. et al. (2001). Estudo a corrosão de ligas 7XXX-T7 em meio salino. Revista Latinoamericana de Metalurgia y Materiales, v. 21 (1).

BIRBILIS, N., CAVANAUGH, M.K., BUCHHEIT, R.G. (2006). Electrochemical behavior and localized corrosion associated with $\mathrm{Al}_{7} \mathrm{Cu} \mathrm{u}_{2} \mathrm{Fe}$ particles in aluminum alloy 7075-T651.

CHEMIN, A. et al. (2013). Influence of $\mathrm{Al}_{7} \mathrm{Cu}_{2} \mathrm{Fe}$ intermetallic particles on the localized corrosion of high strength aluminum alloys. Materials and Design 53, p.118-123.

DAVIS, J.R. Aluminium and Aluminium Alloys. ASM International Materials Park, OH, 784p, ISBN: 087170496X, 1993, p. 494.

De WIT, J. H.W. (2004). Local potential measurements with the SKPFM on aluminium alloys. Electrochimica Acta, v.49, n.17/18, p.2841-2850.

GUILLAUMIN V., MANKOWSKI G (1999). Localized corrosion of 2024 T351 aluminium alloy in chloride media. Corr. Sci., 41(3), p. 421-438.

KIM,Y. et al. (2006). Study of oxygen reduction on stainless steel surfaces and its contribution to acoustic emission recorded during corrosion processes. Corrosion Science, v.48, n. 12, p.3945. 
KLOET J. V., HASSEL A.W., STRATMANN, M (2005). Effect of pretreatment on the intermetallics in aluminum alloy 2024-T3.Z. Phys. Chem.,219 (11), p. 1505-1518.

LANDOLT, D. (2007). Corrosion and surfaces chemistry of metals. Switzerland: EPFL.

MARKOVIC, N. et al. (2001).Oxygen reduction reaction on PT and PT bimetallic surfaces: a selective review. Fuel Cells, v.1, n.2, p.105-116.

MORETO, J. A. (2012). Estudo da corrosão e corrosão-fadiga em ligas de Al e Al- Li de Alta Resistência para Aplicação Aeronáutica. Tese (doutorado), Escola de Engenharia de São Carlos.

NISANCIOGLU, K. (1990). Electrochemical behavior of aluminum-base intermetallics containing iron. J. Electrochem. Soc, 137, p.69-77.

NISANCIOGLU, K. (1992). Corrosion of aluminum alloys. Proceedings of ICAA3, v.3, p. 239-259.

OHTSUKA, T.; NOMURA, N. (1997). The Dependence of the optical property of Ti anodic oxide film on its growth rate by ellipsometry. Corrosion Science, v.39, ,n.7, p.1253-1263.

PAO, P.S. et al. (2003). Corrosion-fatigue crack growth in friction stir welded Al 7050. Scripta Materiala, v.45, n.5, p.605-612.

TOTTEN, G. E., MACKENZIE, D. S. (2003). Handbook of physical metallurgy and process. New York: CRC Press. v.1.

VIEIRA, A. C. et al. (2011).Effect of $\mathrm{Al}_{2} \mathrm{Cu}$ precipitates size and mass transport on the polarisation behavior of age-hardened $\mathrm{Al}-\mathrm{Si}-\mathrm{Cu}-\mathrm{Mg}$ alloys in $0.05 \mathrm{M} \mathrm{NaCl}$. Electrochimica Acta, 56, 3821-3828.

WANG, D., MA, Z.I. (2009). Effect of pre-strain on microstructure and stress corrosion cracking of over-aged7050 aluminium alloy. Journal and Compounds, v. 469, p.445-450.

WLOKA, J., VIRTANEN, S. (2007). Microstructural effects on the corrosion behavior of high strength Al-Zn-Mg-Cu alloys in an averaged condition. Journal of the Electrochemical Society, v.154, n.8, p.C411-C423.

\section{Agradecimentos}

Os autores gostariam de agradecer a Escola de Engenharia de São Carlos - EESC/USP pelo suporte, ao Centro de Tecnologias Mecânicas e de Materiais - CT2M da Universidade do Minho - Portugal pelo uso dos laboratórios e suporte financeiro para a realização dos ensaios eletroquímicos, a CAPES pelo auxílio financeiro disponibilizado através de bolsa de doutorado PDEE (Processo Capes: BEX-4936/10-8) e ao CNPq pela bolsa de IC do aluno José Neto Costa Mota.

Informações complementares dos autores:

José Neto Costa Mota: Aluno do curso de API do Instituto Federal Goiano - Campus Morrinhos - Rodovia BR-153, Km 633, Zona Rural, Caixa Postal 92, CEP: 75659-000 Morrinhos, Goiás, Brasil, e-mail: netocostamota@hotmail.com

Luciana Sgarbi Rossino: Professora da Faculdade de Tecnologia de Sorocaba - Av. Engenheiro Carlos Reinaldo Mendes, 2015, Alto da Boa Vista, CEP: 18013-280, Sorocaba, São Paulo, Brasil, e-mail: 1u-sgarbi@ hotmail.com 
Waldek Wladimir Bose Filho: Professor Titular da Universidade de São Paulo, Departamento de Engenharia de Materiais -Av. Trabalhador São-carlense, 400, Centro, CEP: 13566-590 São Carlos, São Paulo, Brasil, e-mail: waldek@sc.usp.br

Marcelo Ferreira Moreira: Pesquisador do Instituto de Pesquisas Tecnológicas do Estado de São Paulo, Av. Prof. Almeida Prado, 532 - Cidade Universitária Armando de Salles Oliveira LMMC - Prédio 2, CEP: 05508901, São Paulo, Brasil, e-mail: mfmoreir@ipt.br

João Carlos Salvador Fernandes: Professor Associado do Instituto Superior Técnico de Lisboa, Departamento de Engenharia Química - Av. Rovisco, P-1049-001, Lisboa, Portugal, e-mail: joao.salvador@ist.utl.pt

Luís Augusto da Rocha: Professor Adjunto da Universidade Estadual Paulista Júlio de Mesquita Filho, Faculdade de Ciências de Bauru, Departamento de Física - Av. Engenheiro Luiz Edmundo Carrijo Coube, Residencial Presidente Geisel, CEP: 17033-360, Bauru, SP, Brasil. CT2M - Centro de Tecnologias Mecânicas e de Materiais, Universidade do Minho, Campus de Azurém, P-4800-058 Guimarães, Portugal, e-mail: lrocha@dem.uminho.pt 